\title{
Soft b-open sets and soft b-continuous functions
}

\author{
Metin Akdag • Alkan Ozkan
}

Received: 3 March 2014 / Accepted: 17 June 2014/Published online: 17 July 2014

(C) The Author(s) 2014. This article is published with open access at Springerlink.com

\begin{abstract}
In this paper, a new class of generalized soft open sets in soft topological spaces, called soft b-open sets, is introduced and studied. Then discussed the relationships among soft $\alpha$-open sets, soft semi-open sets, soft pre-open sets and soft $\beta$-open sets. We also investigated the concepts of soft b-open functions and soft b-continuous functions and discussed their relations with soft continuous and other weaker forms of soft continuous functions.
\end{abstract}

Keywords Soft b-open (sb-open) sets $\cdot$ Soft b-closed (sb-closed) sets $\cdot$ Soft b-continuous functions

Mathematics Subject Classification (2010) Primary 54C08 $\cdot$ Secondary 54C10

\section{Introduction and preliminaries}

Molodtsov [1] initiated a novel concept of soft set theory, which is a completely new approach for modeling vagueness and uncertainty. He successfully applied the soft set theory into several directions such as smoothness of functions, game theory, Riemann Integration, theory of measurement, and so on. Soft set theory and its applications have shown great development in recent years. This is because of the general nature of parametrization expressed by a soft set. Shabir and Naz [2] introduced the notion of soft topological spaces which are defined over an initial universe with a fixed set of

M. Akdag · A. Ozkan ( $\square)$

Department of Mathematics, Faculty of Science, Cumhuriyet

University, Sivas 58140, Turkey

e-mail: alkan_mat@hotmail.com

M. Akdag

e-mail: makdag@cumhuriyet.edu.tr parameters. Later, Zorlutuna et al.[3], Aygunoglu and Aygun [4] and Hussain et al are continued to study the properties of soft topological space. They got many important results in soft topological spaces. Weak forms of soft open sets were first studied by Chen [5]. He investigated soft semi-open sets in soft topological spaces and studied some properties of it. Arockiarani and Arokialancy are defined soft $\beta$-open sets and continued to study weak forms of soft open sets in soft topological space. Later, Akdag and Ozkan [6] defined soft $\alpha$-open (soft $\alpha$-closed) sets.

In the present paper, we introduce some new concepts in soft topological spaces such as soft b-open sets, soft b-closed sets, soft b-interior, soft b-closure, soft b-continuous functions, soft b-open functions and soft b-closed functions. We also study the relationships among soft continuity [7], soft $\alpha$-continuity [6], soft semi-continuity [8], soft pre-continuity [6], soft $\beta$-continuous [9] and soft b-continuity of functions defined on soft topological spaces. With the help of counter examples we show the non-coincidence of these various types of mappings.

Throughout the paper, the space $X$ and $Y$ stand for soft topological spaces with $((X, \tau, E)$ and $(Y, v, K))$ assumed unless otherwise stated. Moreover, throughout this paper, a soft mapping $f: X \rightarrow Y$ stands for a mapping, where $f:(X, \tau, E) \rightarrow(Y, v, K), u: X \rightarrow Y$ and $p: E \rightarrow K$ are assumed mappings unless otherwise stated.

Definition 1 [1] Let $X$ be an initial universe and $E$ be a set of parameters. Let $P(X)$ denote the power set of $X$ and $A$ be a non-empty subset of $E$. A pair $(F, A)$ is called a soft set over $X$, where $F$ is a mapping given by $F: A \rightarrow P(X)$. In other words, a soft set over $X$ is a parameterized family of subsets of the universe $X$. For $\varepsilon \in A, F(\varepsilon)$ may be considered as the set of $\varepsilon$-approximate elements of the soft set $(F, A)$. 
Definition 2 [10] A soft set $(F, A)$ over $X$ is called a null soft set, denoted by $\Phi$, if $e \in A, F(e)=\emptyset$.

Definition 3 [10] A soft set $(F, A)$ over $X$ is called an absolute soft set, denoted by $\widetilde{A}$, if $e \in A, F(e)=X$.

If $A=E$, then the $A$-universal soft set is called a universal soft set, denoted by $\widetilde{X}$.

Definition 4 [2] Let $Y$ be a non-empty subset of $X$, then $\widetilde{Y}$ denotes the soft set $(Y, E)$ over $X$ for which $Y(e)=Y$, for all $e \in E$.

Definition 5 [10] The union of two soft sets of $(F, A)$ and $(G, B)$ over the common universe $X$ is the soft set $(H, C)$, where $C=A \widetilde{\cup} B$ and for all $e \in C$

$H(e)=\left\{\begin{array}{lll}F(e), & \text { if } & e \in A-B, \\ G(e), & \text { if } \quad e \in B-A, \\ F(e) \cup G(e), & \text { if } \quad e \in A \cap B .\end{array}\right.$

We write $(F, A) \widetilde{\cup}(G, B)=(H, C)$.

Definition $6[10]$ The intersection $(H, C)$ of two soft sets $(F, A)$ and $(G, B)$ over a common universe $X$, denoted $(F, A) \widetilde{\cap}(G, B)$, is defined as $C=A \cap B$, and $H(e)=$ $F(e) \cap G(e)$ for all $e \in C$.

Definition 7 [10] Let $(F, A)$ and $(G, B)$ be two soft sets over a common universe $X$. $(F, A) \widetilde{\subset}(G, B)$, if $A \subset B$, and $H(e)=F(e) \subset G(e)$ for all $e \in A$.

Definition 8 [2] Let $\tau$ be the collection of soft sets over $X$, then $\tau$ is said to be a soft topology on $X$ if satisfies the following axioms.

(1) $\Phi, \tilde{X}$ belong to $\tau$,

(2) the union of any number of soft sets in $\tau$ belongs to $\tau$,

(3) the intersection of any two soft sets in $\tau$ belongs to $\tau$.

The triplet $(X, \tau, E)$ is called a soft topological space over $X$. Let $(X, \tau, E)$ be a soft topological space over $X$, then the members of $\tau$ are said to be soft open sets in $X$. A soft set $(F, A)$ over $X$ is said to be a soft closed set in $X$, if its relative complement $(F, A)^{c}$ belongs to $\tau$.

Definition 9 [11] For a soft set $(F, A)$ over $X$, the relative complement of $(F, A)$ is denoted by $(F, A)^{c}$ and is defined by $(F, A)^{c}=\left(F^{c}, A\right)$, where $F^{c}: A \rightarrow P(X)$ is a mapping given by $F^{c}(\alpha)=X-F(\alpha)$ for all $\alpha \in A$.

\section{Soft b-open sets}

In this section we introduce soft b-open sets in soft topological spaces and study some of their properties.

Definition 10 A soft set $(F, A)$ in a soft topological space $X$ is called (i) soft b-open (sb-open) set iff $(F, A) \widetilde{C}$ int $(\operatorname{cl}((\mathrm{F}, \mathrm{A}))) \widetilde{\cup} \mathrm{cl}(\operatorname{int}((\mathrm{F}, \mathrm{A})))$

(ii) soft b-closed (sb-closed) set iff $(F, A) \widetilde{\supset}$ int $(\mathrm{cl}((\mathrm{F}, \mathrm{A}))) \widetilde{\cap} \mathrm{cl}(\operatorname{int}((\mathrm{F}, \mathrm{A})))$.

Theorem 1 For a soft set $(F, A)$ in a soft topological space $X$

(i) $(F, A)$ is a soft b-open set iff $(F, A)^{c}$ is a soft b-closed set.

(ii) $(F, A)$ is a soft b-closed set iff $(F, A)^{c}$ is a soft b-open set.

Proof Obvious from the Definition 10.

Definition 11 Let $(X, \tau, E)$ be a soft topological space and $(F, A)$ be a soft set over $X$.

(i) Soft b-closure of a soft set $(F, A)$ in $X$ is denoted by $\operatorname{sbcl}((\mathrm{F}, \mathrm{A}))=\tilde{\cap}\{(\mathrm{F}, \mathrm{E}) \widetilde{\supset}(\mathrm{F}, \mathrm{A}):(\mathrm{F}, \mathrm{E})$

is a soft $\mathrm{b}$-closed set of $\mathrm{X}$ \}.

(ii) Soft b-interior of a soft set $(F, A)$ in $X$ is denoted by $\operatorname{sbint}((\mathrm{F}, \mathrm{A}))=\widetilde{\cup}\{(\mathrm{O}, \mathrm{A}) \widetilde{C}(\mathrm{~F}, \mathrm{~A}):(\mathrm{O}, \mathrm{A})$ is a soft $\mathrm{b}$-open set of $\mathrm{X}\}$.

Clearly $\operatorname{sbcl}((\mathrm{F}, \mathrm{A}))$ is the smallest soft b-closed set over $X$ which contains $(F, A)$ and $\operatorname{sbint}((\mathrm{F}, \mathrm{A}))$ is the largest soft b-open set over $X$ which is contained in $(F, A)$.

Theorem 2 Let $(F, A)$ be any soft set a in soft topological space $X$. Then,

(i) $\operatorname{sbcl}\left((\mathrm{F}, \mathrm{A})^{\mathrm{c}}\right)=\widetilde{\mathrm{X}}-\operatorname{sbint}((\mathrm{F}, \mathrm{A}))$.

(ii) $\operatorname{sbint}\left((\mathrm{F}, \mathrm{A})^{\mathrm{c}}\right)=\widetilde{\mathrm{X}}-\operatorname{sbcl}((\mathrm{F}, \mathrm{A}))$.

Proof (i) Let sb-open set $(O, A) \widetilde{\subset}(F, A)$ and sb-closed set $(F, E) \widetilde{\supset}(F, A)^{c}$. Then, $\operatorname{sbint}((\mathrm{F}, \mathrm{A}))=\widetilde{\cup}\left\{(\mathrm{F}, \mathrm{E})^{\mathrm{c}}:(\mathrm{F}, \mathrm{E})\right.$ is a softb - closed set and $\left.(\mathrm{F}, \mathrm{E}) \widetilde{\supset}(\mathrm{F}, \mathrm{A})^{\mathrm{c}}\right\}=\widetilde{\mathrm{X}}-\tilde{\cap}$ $\{(F, E):(F, E) \quad$ is a sb-closed set and $(F, E) \widetilde{\supset}$ $\left.(F, A)^{c}\right\}=\widetilde{X}-\operatorname{sbcl}\left((\mathrm{F}, \mathrm{A})^{\mathrm{c}}\right)$.

Therefore, $\operatorname{sbcl}\left((\mathrm{F}, \mathrm{A})^{\mathrm{c}}\right)=\widetilde{\mathrm{X}}-\operatorname{sbint}((\mathrm{F}, \mathrm{A}))$.

(ii) Let $(O, A)$ be sb-open set.

Then, for a sb-closed set $(O, A) \widetilde{\supset}(F, A),(O, A) \widetilde{C}$ $(F, A)^{c}$.

$\operatorname{sbcl}((\mathrm{F}, \mathrm{A}))=\widetilde{\cap}\left\{(\mathrm{O}, \mathrm{A})^{\mathrm{c}}:(\mathrm{O}, \mathrm{A})\right.$ is a sb - open set and $\left.(\mathrm{O}, \mathrm{A}) \widetilde{C}(\mathrm{~F}, \mathrm{~A})^{\mathrm{c}}\right\}$

$=\widetilde{X}-\widetilde{\cup}\left\{(O, A):(O, A)\right.$ is a sb - openset and $\left.(\mathrm{O}, \mathrm{A}) \widetilde{C}(\mathrm{~F}, \mathrm{~A})^{\mathrm{c}}\right\}$

$=\widetilde{X}-\operatorname{sint}\left((\mathrm{F}, \mathrm{A})^{\mathrm{c}}\right)$.

Therefore, $\operatorname{sbint}\left((\mathrm{F}, \mathrm{A})^{\mathrm{c}}\right)=\widetilde{\mathrm{X}}-\operatorname{sbcl}((\mathrm{F}, \mathrm{A}))$.

Definition 12 Let $(X, \tau, E)$ be a soft topological space over $X$ and $(F, A)$ be a soft set over $X$.

(i) [3] The soft interior of $(F, A)$ is the soft set $\operatorname{int}((\mathrm{F}, \mathrm{A}))=\widetilde{\cup}\{(\mathrm{O}, \mathrm{A}) \widetilde{C}(\mathrm{~F}, \mathrm{~A}):(\mathrm{O}, \mathrm{A})$ is soft open of $X\}$; 
(ii) [2] The soft closure of $(F, A)$ is the soft set $\operatorname{cl}((\mathrm{F}, \mathrm{A}))=\widetilde{\cap}\{(\mathrm{F}, \mathrm{E}) \widetilde{\supset}(\mathrm{F}, \mathrm{A}):(F, E)$ is soft closed of $X$;

(iii) [5] The soft semi-interior of $(F, A)$ is a soft set $\operatorname{sint}((\mathrm{F}, \mathrm{A}))=\widetilde{\cup}\{(O, A) \widetilde{C}(F, A):(O, A)$ is soft semi-open of $X$ \}.

(iv) [5] The soft semi-closure of $(F, A)$ is a soft set $\operatorname{sscl}((F, A))=\widetilde{\cap}\{(F, E) \widetilde{\supset}(F, A):(F, E)$ is soft semi-closed of $X\}$;

The following concepts are used in the sequel.

Definition 13 A soft set $(F, A)$ in a soft topological space $X$ is called

(i) soft regular open (soft regular closed) set [12] if $(F, A)=\operatorname{cl}(\operatorname{int}(\mathrm{F}, \mathrm{A}))(\operatorname{int}(\mathrm{cl}(\mathrm{F}, \mathrm{A}))=(\mathrm{F}, \mathrm{A}))$.

(ii) $\quad$ soft $\alpha$-open (soft $\alpha$-closed) set [6] if $(F, A) \widetilde{C}$ $\operatorname{int}(\operatorname{cl}(\operatorname{int}(\mathrm{F}, \mathrm{A})))(\operatorname{cl}(\operatorname{int}(\mathrm{cl}(\mathrm{F}, \mathrm{A}))) \widetilde{\subset}(F, A))$.

(iii) soft pre-open (soft pre-closed) set [12] if $(F, A) \widetilde{C} \operatorname{int}(\operatorname{cl}(\mathrm{F}, \mathrm{A}))(\operatorname{cl}(\operatorname{int}(\mathrm{F}, \mathrm{A})) \widetilde{C}(\mathrm{~F}, \mathrm{~A}))$.

(iv) soft semi-open (soft semi-closed) set [5] if $(F, A) \widetilde{C} \operatorname{cl}(\operatorname{int}(\mathrm{F}, \mathrm{A}))(\operatorname{int}(\mathrm{cl}(\mathrm{F}, \mathrm{A})) \widetilde{\subset}(\mathrm{F}, \mathrm{A}))$.

(v) soft $\beta$-open (soft $\beta$-closed) set [12] if $(F, A) \widetilde{C} \operatorname{cl}(\operatorname{int}(\mathrm{cl}(\mathrm{F}, \mathrm{A})))(\operatorname{int}(\mathrm{cl}(\operatorname{int}(\mathrm{F}, \mathrm{A})))$ $\widetilde{\subset}(F, A))$.

Lemma 1 Let $(F, A)$ be a soft set in a soft topological space $X$. Then

(i) $\operatorname{sscl}((\mathrm{F}, \mathrm{A}))=(\mathrm{F}, \mathrm{A}) \widetilde{\cup i n t}(\operatorname{cl}((\mathrm{F}, \mathrm{A})))$ and $\operatorname{sint}((\mathrm{F}, \mathrm{A}))=(\mathrm{F}, \mathrm{A}) \widetilde{\cap} \mathrm{cl}(\operatorname{int}((\mathrm{F}, \mathrm{A})))$.

(ii) $\operatorname{spcl}((\mathrm{F}, \mathrm{A}))=(\mathrm{F}, \mathrm{A}) \widetilde{\cup} \operatorname{cl}(\operatorname{int}((\mathrm{F}, \mathrm{A})))$ and $\operatorname{spint}((\mathrm{F}, \mathrm{A}))=(\mathrm{F}, \mathrm{A}) \tilde{\operatorname{nint}}(\mathrm{cl}((\mathrm{F}, \mathrm{A})))$.

Proof (i)

$\operatorname{sscl}((\mathrm{F}, \mathrm{A})) \tilde{\supset} \operatorname{int}(\operatorname{cl}(\operatorname{sscl}((\mathrm{F}, \mathrm{A})))) \widetilde{\operatorname{int}}(\mathrm{cl}((\mathrm{F}, \mathrm{A})))$

$(F, A) \widetilde{\cup} \operatorname{sscl}((\mathrm{F}, \mathrm{A}))=\operatorname{sscl}((\mathrm{F}, \mathrm{A})) \widetilde{\supset}(\mathrm{F}, \mathrm{A}) \widetilde{\cup} \operatorname{int}(\operatorname{cl}((\mathrm{F}, \mathrm{A})))$

$\operatorname{so}(\mathrm{F}, \mathrm{A}) \widetilde{\cup} \operatorname{int}(\mathrm{cl}((\mathrm{F}, \mathrm{A}))) \widetilde{\subset} \operatorname{sscl}((\mathrm{F}, \mathrm{A}))$.

Also

$$
\begin{aligned}
& (F, A) \widetilde{\subset} \operatorname{sscl}((\mathrm{F}, \mathrm{A})) \\
& \operatorname{int}(\operatorname{cl}((\mathrm{F}, \mathrm{A}))) \widetilde{\subset} \operatorname{int}(\operatorname{cl}(\operatorname{sscl}((\mathrm{F}, \mathrm{A})))) \widetilde{\subset} \operatorname{sscl}((\mathrm{F}, \mathrm{A})) \\
& (F, A) \widetilde{\cup} \operatorname{int}(\mathrm{cl}((\mathrm{F}, \mathrm{A}))) \widetilde{\subset} \operatorname{sscl}((\mathrm{F}, \mathrm{A})) \widetilde{\cup}(\mathrm{F}, \mathrm{A}) \widetilde{C} \operatorname{sscl}((\mathrm{F}, \mathrm{A})) .
\end{aligned}
$$

Hence from 1 and $2, \operatorname{sscl}((\mathrm{F}, \mathrm{A}))=(\mathrm{F}, \mathrm{A}) \widetilde{\cup} \operatorname{int}(\mathrm{cl}((\mathrm{F}, \mathrm{A})))$.

(ii) Similar by taking the complements.

Lemma 2 In a soft topological space we have the following (i) Every soft regular open set is soft open.

(ii) Every soft open set is soft $\alpha$-open.

(iii) Every soft $\alpha$-open set is both soft semi-open and soft pre-open.

(iv) Every soft semi-open set and every soft pre-open set is soft $\beta$-open.

Let $X$ be a soft topological space.Then, the family of all soft regular open (resp. soft $\alpha$-open, soft semi-open, soft pre-open, soft $\beta$-open) sets in $X$ may be denoted by sr (resp. sa-open, ss-open, sp-open, $s \beta$-open) sets. The complement of the above represents the family of soft regular closed (resp. soft $\alpha$-closed, soft semi-closed, soft pre-closed, soft $\beta$-closed) sets in $X$ and may be denoted by $\mathrm{sr}$ (resp. s $\alpha$ closed, ss-closed, sp-closed, s $\beta$-closed) sets. Also, the family of all soft b-open (resp. soft b-closed) sets in $X$ may be denoted by $\operatorname{SbOS}(\mathrm{X})$ (resp. SbCS(X)).

Theorem 3 In a soft topological space $X$

(i) Every sp-open set is sb-open set.

(ii) Every ss-open set is sb-open set.

Proof (i) Let $(F, A)$ be a sp-open set in a soft topological space $X$.

Then, $(F, A) \widetilde{\subset} \operatorname{int}(\operatorname{cl}((\mathrm{F}, \mathrm{A})))$ which implies

$(F, A) \widetilde{\subset} \operatorname{int}(\operatorname{cl}((\mathrm{F}, \mathrm{A}))) \widetilde{\cup} \operatorname{int}((\mathrm{F}, \mathrm{A})) \widetilde{\subset} \operatorname{int}(\operatorname{cl}((\mathrm{F}, \mathrm{A})))$ $\widetilde{U} \operatorname{cl}(\operatorname{int}((\mathrm{F}, \mathrm{A})))$.

Thus $(F, A)$ is sb-open set.

(ii) Let $(F, A)$ be a ss-open set in a soft topological space $X$. Then, $(F, A) \widetilde{C} \mathrm{cl}(\operatorname{int}((\mathrm{F}, \mathrm{A})))$ which implies $(F, A) \widetilde{\subset} \mathrm{cl}$ $(\operatorname{int}((\mathrm{F}, \mathrm{A}))) \widetilde{\cup} \operatorname{int}((\mathrm{F}, \mathrm{A})) \widetilde{C} \operatorname{cl}(\operatorname{int}((\mathrm{F}, \mathrm{A}))) \widetilde{\cup} \operatorname{int}(\mathrm{cl}((\mathrm{F}, \mathrm{A})))$. Thus $(F, A)$ is sb-open set.

The converses are not true as seen in the following example:

Example 1 Let $X=\left\{h_{1}, h_{2}, h_{3}, h_{4}\right\}, E=\left\{e_{1}, e_{2}, e_{3}\right\}$ and $\tau=\left\{\emptyset, \widetilde{X},\left(F_{1}, E\right),\left(F_{2}, E\right),\left(F_{3}, E\right), \ldots,\left(F_{15}, E\right)\right\}$, where $\left(F_{1}, E\right),\left(F_{2}, E\right),\left(F_{3}, E\right), \ldots\left(F_{15}, E\right)$ are soft sets over $X$, defined as follows:

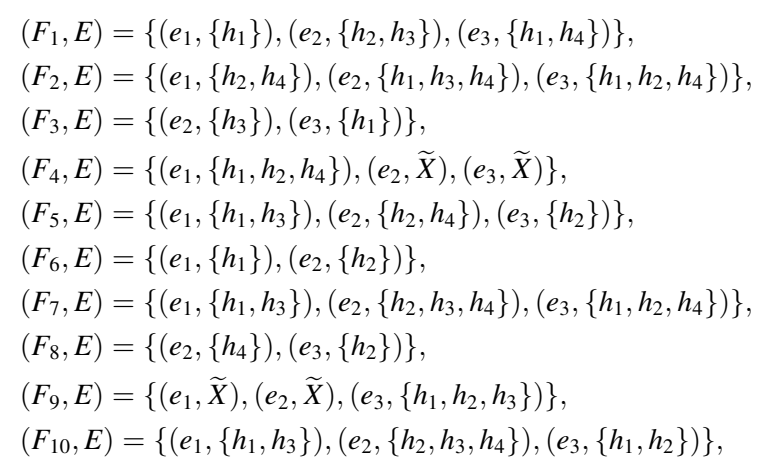


$\left(F_{11}, E\right)=\left\{\left(e_{1},\left\{h_{2}, h_{3}, h_{4}\right\}\right),\left(e_{2}, \widetilde{X}\right),\left(e_{3},\left\{h_{1}, h_{2}, h_{3}\right\}\right)\right\}$,

$\left(F_{12}, E\right)=\left\{\left(e_{1},\left\{h_{1}\right\}\right),\left(e_{2},\left\{h_{2}, h_{3}, h_{4}\right\}\right),\left(e_{3},\left\{h_{1}, h_{2}, h_{4}\right\}\right)\right\}$,

$\left(F_{13}, E\right)=\left\{\left(e_{1},\left\{h_{1}\right\}\right),\left(e_{2},\left\{h_{2}, h_{4}\right\}\right),\left(e_{3},\left\{h_{2}\right\}\right)\right\}$,

$\left(F_{14}, E\right)=\left\{\left(e_{1},\left\{h_{3}, h_{4}\right\}\right),\left(e_{2},\left\{h_{1}, h_{2}\right\}\right)\right\}$,

$\left(F_{15}, E\right)=\left\{\left(e_{1},\left\{h_{1}\right\}\right),\left(e_{2},\left\{h_{2}, h_{3}\right\}\right),\left(e_{3},\left\{h_{1}\right\}\right)\right\}$.

Then, $\tau$ defines a soft topology on $X$, and thus $(X, \tau, E)$ is a soft topological space over $X$. Clearly, the soft closed sets are $\widetilde{X}, \emptyset,\left(F_{1}, E\right)^{c},\left(F_{2}, E\right)^{c},\left(F_{3}, E\right)^{c}, \ldots,\left(F_{15}, E\right)^{c}$.

Then, let us take $(F, A)=\left\{\left(e_{1},\left\{h_{2}, h_{4}\right\}\right), \quad\left(e_{2},\left\{h_{1}\right.\right.\right.$, $\left.\left.\left.h_{3}\right\}\right),\left(e_{3},\left\{h_{1}, h_{3}, h_{4}\right\}\right)\right\} ;$ then $\operatorname{int}(\operatorname{cl}((\mathrm{F}, \mathrm{A}))) \widetilde{U} \operatorname{cl}(\operatorname{int}((\mathrm{F}$, $A)))=\widetilde{X}$, and so $(F, A) \widetilde{\subset} \operatorname{int}(\operatorname{cl}((\mathrm{F}, \mathrm{A}))) \widetilde{\cup} \operatorname{cl}(\operatorname{int}((\mathrm{F}, \mathrm{A})))$; hence, $(F, A)$ is sb-open set, but not sp-open set (since $(F, A)$ is not sp-open set).

Now, let us take $(G, E)=\left\{\left(e_{1},\left\{h_{4}\right\}\right),\left(e_{2},\left\{h_{1}, h_{2}\right.\right.\right.$, $\left.\left.\left.h_{3}\right\}\right),\left(e_{3},\left\{h_{2}, h_{4}\right\}\right)\right\} ; \quad$ then $\operatorname{int}(\operatorname{cl}((\mathrm{G}, \mathrm{E}))) \quad \widetilde{\cup} \operatorname{cl}(\operatorname{int}((\mathrm{G}$, $E)))=\widetilde{X}$, and so $(G, E) \widetilde{C} \operatorname{int}(\operatorname{cl}((\mathrm{G}, \mathrm{E}))) \widetilde{\cup} \mathrm{cl} \quad \operatorname{int}((\mathrm{G}$, $E)))$; thus, $(G, E)$ is sb-open set, but not ss-open set.

\section{Remark 1}

(i) If $(F, A)$ is a soft set of soft topological space $X$, then $\operatorname{sbcl}((\mathrm{F}, \mathrm{A}))$ is the smallest sb-closed set containing $(F, A)$.

Thus, $\operatorname{sbcl}(\mathrm{F}, \mathrm{A})=(\mathrm{F}, \mathrm{A}) \widetilde{\cup}[\operatorname{int}(\mathrm{cl}((\mathrm{F}, \mathrm{A}))) \widetilde{n} \mathrm{cl}($ int $((F, A)))]$.

(ii) If $(F, A)$ is a soft set of soft topological space $X$ then $\operatorname{sbint}((\mathrm{F}, \mathrm{A}))$ is the largest sb-open set contained in $(F, A)$.

Thus, $\operatorname{sbint}(\mathrm{F}, \mathrm{A})=(\mathrm{F}, \mathrm{A}) \widetilde{\cap}[\operatorname{int}(\mathrm{cl}((\mathrm{F}, \mathrm{A}))) \widetilde{U} \mathrm{cl}($ int $((F, A)))]$.

Theorem 4 In a soft topological space $X$, every sb-open (sb-closed) set is $\mathrm{s} \beta$-open ( $\mathrm{s} \beta$-closed) set.

Proof Let $(F, A)$ be a sb-open set in $X$.

Then $(F, A) \widetilde{\subset} \operatorname{cl}(\operatorname{int}((\mathrm{F}, \mathrm{A}))) \widetilde{\cup} \operatorname{int}(\operatorname{cl}((\mathrm{F}, \mathrm{A})))$

$\widetilde{C} \operatorname{cl}(\operatorname{int}(\mathrm{cl}((\mathrm{F}, \mathrm{A})))) \widetilde{\cup} \operatorname{int}(\mathrm{cl}((\mathrm{F}, \mathrm{A}))) \widetilde{C} \operatorname{cl}(\operatorname{int}(\mathrm{cl}$

$((F, A))))$.

As a result $(F, A)$ is $\mathrm{s} \beta$-open set.

The converse is not true as seen in the following example:

Example 2 Let $X=\left\{h_{1}, h_{2}, h_{3}, h_{4}\right\}, E=\left\{e_{1}, e_{2}, e_{3}\right\}$ and let $(X, \tau, E)$ be soft topological space over $X$. Let us consider the soft topology $\tau$ on $X$ given in Example 1; i.e., $\tau=\left\{\emptyset, \widetilde{X},\left(F_{1}, E\right),\left(F_{2}, E\right),\left(F_{3}, E\right), \ldots,\left(F_{15}, E\right)\right\}$.

Then, let us take $(H, E)=\left\{\left(e_{1},\left\{h_{1}, h_{2}\right\}\right),\left(e_{2},\left\{h_{3}, h_{4}\right\}\right)\right.$, $\left.\left(e_{3},\left\{h_{1}, h_{3}, h_{4}\right\}\right)\right\} ; \quad$ then $\operatorname{cl}(\operatorname{int}(\operatorname{cl}((\mathrm{H}, \mathrm{E}))))=\left\{\left(\mathrm{e}_{1}, \widetilde{\mathrm{X}}\right)\right.$, $\left.\left(e_{2},\left\{h_{1}, h_{3}, h_{4}\right\}\right),\left(e_{3}, \widetilde{X}\right)\right\}$, and so $(H, E) \widetilde{C l} \operatorname{cl}(\operatorname{int}(\mathrm{cl}((\mathrm{H}$, $E))))$; therefore, $(H, E)$ is $\mathrm{s} \beta$-open set but not $\mathrm{s} b$-open set.
Remark 2 From the above theorems, we have the following

soft regular set $\rightarrow$ soft open set

soft $\alpha$-open set

soft semi-open set

soft pre-open set

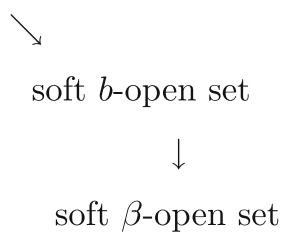

Theorem 5 In a soft topological space $X$

(i) An arbitrary union of sb-open sets is a sb-open set.

(ii) An arbitrary intersection of sb-closed sets is a sbclosed set.

Proof (i) Let $\left\{(F, A)_{\alpha}\right\}$ be a collection of sb-open sets. Then, for each $\alpha$,

$$
\begin{aligned}
&(F, A)_{\alpha} \widetilde{\subset} \operatorname{cl}\left(\operatorname{int}\left((\mathrm{F}, \mathrm{A})_{\alpha}\right)\right) \widetilde{\cup} \operatorname{int}\left(\operatorname{cl}\left((\mathrm{F}, \mathrm{A})_{\alpha}\right)\right) \text {. Now } \\
& \widetilde{\cup}\left((F, A)_{\alpha}\right) \widetilde{\subset} \widetilde{\cup}\left[\operatorname{cl}\left(\operatorname{int}\left((\mathrm{F}, \mathrm{A})_{\alpha}\right)\right) \widetilde{\cup} \operatorname{cl}\left(\operatorname{int}\left((\mathrm{F}, \mathrm{A})_{\alpha}\right)\right)\right] \\
&\left.\left.\widetilde{\mathcal{C}}\left[\operatorname{cl}\left(\operatorname{int}\left(\widetilde{\cup}\left((\mathrm{F}, \mathrm{A})_{\alpha}\right)\right)\right)\right) \widetilde{\cup} \operatorname{int}\left(\operatorname{cl}\left(\widetilde{\cup}\left((\mathrm{F}, \mathrm{A})_{\alpha}\right)\right)\right)\right)\right] .
\end{aligned}
$$

Hence $\widetilde{\cup}\left((F, A)_{\alpha}\right)$ is a sb-open set.

(ii) Similarly by taking complements.

Theorem 6 In a soft topological space $X,(F, A)$ is sbclosed ( $b$-open) set if and only if $(F, A)=\operatorname{sbcl}(\mathrm{F}, \mathrm{A})$ $((F, A)=\operatorname{sbint}(\mathrm{F}, \mathrm{A}))$.

Proof Suppose $(F, A)=\operatorname{sbcl}((\mathrm{F}, \mathrm{A}))=\widetilde{\cap}\{(F, E)$ : $(F, E)$ is a sb-closed set and $(F, E) \widetilde{\supset}(F, A)\}$ that implies, $(F, A) \in \widetilde{\cap}\{(F, E)$ is a sb-closed set and $(F, E) \widetilde{\supset}(F, A)\}$, that implies $(F, A)$ is sb-closed set.

Conversely, suppose $(F, A)$ is a sb-closed set in $X$.

We take $(F, A) \widetilde{\subset}(F, A)$ and $(F, A)$ is a sb-closed. Therefore,

$(F, A) \in \widetilde{\cap}\{(F, E):(F, E)$ is a s b-closed set and $(F, E)$ $\widetilde{\supset}(F, A)\}$.

$(F, A) \widetilde{C}(F, E)$ implies, $(F, A)=\widetilde{\cap}\{(F, E):(F, E)$ is a sb-closed set and $(F, E) \widetilde{\supset}(F, A)\}=\operatorname{sbcl}((\mathrm{F}, \mathrm{A}))$.

For $(F, A)=\operatorname{sbint}((\mathrm{F}, \mathrm{A}))$ we apply soft interiors.

Theorem 7 In a soft topological space $X$ the following hold for sb-closure.

(i) $\operatorname{sbcl}(\Phi)=\Phi$.

(ii) $\operatorname{sbint}(\Phi)=\Phi$. 
(iii) $\operatorname{sbcl}(\mathrm{F}, \mathrm{A})$ is a sb-closed set in $X$.

(iv) $\operatorname{sbcl}(\operatorname{sbcl}(\mathrm{F}, \mathrm{A}))=\operatorname{sbcl}((\mathrm{F}, \mathrm{A}))$.

Proof The proof is obvious.

Theorem 8 In a soft topological space $X$ the following relations hold;

(i) $\operatorname{sbcl}((\mathrm{F}, \mathrm{A}) \widetilde{\cup}(\mathrm{F}, \mathrm{B})) \widetilde{\supset} \operatorname{sbcl}((\mathrm{F}, \mathrm{A})) \widetilde{\cup} \operatorname{sbcl}((\mathrm{F}, \mathrm{B}))$,

(ii) $\operatorname{sbcl}((\mathrm{F}, \mathrm{A}) \widetilde{\cap}(\mathrm{F}, \mathrm{A})) \widetilde{\subset} \operatorname{sbcl}((\mathrm{F}, \mathrm{A})) \widetilde{\cap} \operatorname{sbcl}((\mathrm{F}, \mathrm{B}))$.

Proof (i) $(F, A) \widetilde{\subset}(F, A) \widetilde{\cup}(F, B)$ or $(F, B) \widetilde{\subset}(F, A) \widetilde{\cup}(\mathrm{F}, \mathrm{B})$ that implies $\operatorname{sbcl}((\mathrm{F}, \mathrm{A})) \widetilde{\subset} \operatorname{sbcl} \quad((F, A) \widetilde{\cup}(F, B))$ or $\operatorname{sbcl}((\mathrm{F}, \mathrm{B})) \widetilde{\subset} \operatorname{sbcl}((\mathrm{F}, \mathrm{A}) \widetilde{\cup}(\mathrm{F}, \mathrm{B}))$.

Thus, $\quad \operatorname{sbcl}((\mathrm{F}, \mathrm{A}) \widetilde{\cup}(\mathrm{F}, \mathrm{B})) \widetilde{\supset} \operatorname{sbcl}((\mathrm{F}, \mathrm{A})) \widetilde{\cup} \operatorname{sbcl}((\mathrm{F}, \mathrm{B}))$. (ii) Similar to that of (i).

Theorem 9 In a soft topological space $X$ the following relations hold;

(i) $\operatorname{sbint}((\mathrm{F}, \mathrm{A}) \widetilde{\cup}(\mathrm{F}, \mathrm{B})) \widetilde{\supset} \operatorname{sbint}((\mathrm{F}, \mathrm{A})) \widetilde{U} \operatorname{sbint}((\mathrm{F}, \mathrm{B}))$,

(ii) $\operatorname{sbint}((\mathrm{F}, \mathrm{A}) \widetilde{\cap}(\mathrm{F}, \mathrm{B})) \widetilde{\subset} \operatorname{sbint}((\mathrm{F}, \mathrm{A})) \widetilde{\cap} \operatorname{sbint}((\mathrm{F}, \mathrm{B}))$.

Proof (i) $(F, A) \widetilde{\subset}(F, A) \widetilde{\cup}(F, B)$ or $(F, B) \widetilde{\subset}(F, A) \widetilde{\cup}(F, B)$ that implies $\operatorname{sbint}((\mathrm{F}, \mathrm{A})) \widetilde{\subset} \operatorname{sbint}((\mathrm{F}, \mathrm{A}) \widetilde{\cup}(\mathrm{F}, \mathrm{B}))$ or sbint $((F, B)) \widetilde{\subset} \operatorname{sbint}((\mathrm{F}, \mathrm{A}) \widetilde{\cup}(\mathrm{F}, \mathrm{B}))$.

Thus, $\operatorname{sbint}((\mathrm{F}, \mathrm{A}) \widetilde{\cup}(\mathrm{F}, \mathrm{B})) \widetilde{\supset} \operatorname{sbint}((\mathrm{F}, \mathrm{A})) \widetilde{\cup} \operatorname{sbint}$ $((F, B))$. (ii) Similar to that of (i).

Theorem 10 Let $(F, A)$ be a sb-open set in a soft topological space $X$.

(i) If $(F, A)$ is a sr-closed set then $(F, A)$ is a sp-open set.

(ii) If $(F, A)$ is a sr-open set then $(F, A)$ is a ss-open set.

Proof Since $(F, A)$ is sb-open set, $(F, A) \widetilde{C} \operatorname{cl}(\operatorname{int}((\mathrm{F}, \mathrm{A})))$ $\widetilde{U} \operatorname{int}(\operatorname{cl}((\mathrm{F}, \mathrm{A})))$,

(i) Now let $(F, A)$ be sr-closed set. Therefore, $(F, A)=\operatorname{cl}(\operatorname{int}((\mathrm{F}, \mathrm{A})))$.

Then $(F, A) \widetilde{C}(F, A) \widetilde{\cup} \operatorname{int}(\operatorname{cl}((\mathrm{F}, \mathrm{A})))$. That implies $(F, A) \widetilde{C} \operatorname{int}(\operatorname{cl}((\mathrm{F}, \mathrm{A})))$.

Hence $(F, A)$ is sp-open set.

(ii) Let $(F, A)$ be sr-open set. Therefore, $(F, A)=$ int $(\operatorname{cl}((\mathrm{F}, \mathrm{A})))$.

Then $(F, A) \widetilde{\subset}(F, A) \widetilde{\cup} \operatorname{cl}(\operatorname{int}((\mathrm{F}, \mathrm{A})))$.

That implies $(F, A) \widetilde{C} \operatorname{cl}(\operatorname{int}((\mathrm{F}, \mathrm{A})))$. Thus $(F, A)$ is ssopen set.

Theorem 11 Let $(F, A)$ be a sb-open set in a soft topological space $X$.

(i) If $(F, A)$ is a sr-closed set then $(F, A)$ is a ss-closed set. (ii) If $(F, A)$ is a sr-open set then $(F, A)$ is a sp-closed set.

Theorem 12 For any sb-open set $(F, A)$ in soft topological space $X, \operatorname{cl}((\mathrm{F}, \mathrm{A}))$ is sr-closed set.

Proof Let $(F, A)$ be sb-open set in $X$ which implies $(F, A)$ $\widetilde{\subset} \operatorname{cl}(\operatorname{int}((\mathrm{F}, \mathrm{A}))) \widetilde{\cup} \operatorname{int}(\operatorname{cl}((\mathrm{F}, \mathrm{A})))$. Therefore, $\operatorname{cl}(\mathrm{F}, \mathrm{A}) \widetilde{\subset} \mathrm{cl}$ $(\operatorname{cl}(\operatorname{int}((\mathrm{F}, \mathrm{A})))) \widetilde{\cup} \operatorname{cl}(\operatorname{int}(\operatorname{cl}((\mathrm{F}, \mathrm{A})))) \widetilde{\subset} \operatorname{cl}(\operatorname{int}(\operatorname{cl}((\mathrm{F}, \mathrm{A}))))$.

Also $\operatorname{cl}(\operatorname{int}(\operatorname{cl}((\mathrm{F}, \mathrm{A})))) \widetilde{\subset} \operatorname{cl}((\mathrm{F}, \mathrm{A}))$. Thus $\operatorname{cl}(\operatorname{int}(\mathrm{cl}$ $((F, A))))=\operatorname{cl}((\mathrm{F}, \mathrm{A}))$.

So $\operatorname{cl}((\mathrm{F}, \mathrm{A}))$ is sr-closed set.

Theorem 13 For any sb-closed set $(F, A)$ in a soft topological space $X$. $\operatorname{int}((\mathrm{F}, \mathrm{A}))$ is sr-open set.

Proof Let $(F, A)$ be sb-closed set in $X$ which implies $\operatorname{cl}(\operatorname{int}((\mathrm{F}, \mathrm{A}))) \widetilde{\cap} \operatorname{int}(\operatorname{cl}((\mathrm{F}, \mathrm{A}))) \widetilde{\subset}(\mathrm{F}, \mathrm{A})$. Thus

$\operatorname{int}(\operatorname{cl}(\operatorname{int}((\mathrm{F}, \mathrm{A})))) \widetilde{\operatorname{int}}(\operatorname{int}(\mathrm{cl}((\mathrm{F}, \mathrm{A})))) \widetilde{\subset} \operatorname{int}((\mathrm{F}, \mathrm{A}))$.

$\operatorname{int}(\operatorname{cl}(\operatorname{int}((\mathrm{F}, \mathrm{A})))) \widetilde{\widetilde{i n t}}((\mathrm{~F}, \mathrm{~A}))$

Also

$\operatorname{int}((\mathrm{F}, \mathrm{A})) \widetilde{\subset} \operatorname{int}(\operatorname{cl}(\operatorname{int}((\mathrm{F}, \mathrm{A}))))$.

Thus from (3) and (4) $\operatorname{int}((\mathrm{F}, \mathrm{A}))=\operatorname{int}(\operatorname{cl}(\operatorname{int}((\mathrm{F}, \mathrm{A}))))$. So $\operatorname{int}((\mathrm{F}, \mathrm{A}))$ is sr-open set.

Theorem 14 Let $(F, A)$ be a sb-open (sb-closed) set in a soft topological space $X$, such that $\operatorname{int}((\mathrm{F}, \mathrm{A}))=\Phi$. Then, $(F, A)$ is a sp-open set.

Theorem 15 Let $(F, A)$ be a sb-open (sb-closed) set in a soft topological space $X$, such that $\operatorname{cl}((\mathrm{F}, \mathrm{A}))=\Phi$. Then, $(F, A)$ is a ss-open set.

Theorem 16 Let $(F, A)$ be a set of a soft topological space $X$. Then,

(i) $\operatorname{sbcl}((\mathrm{F}, \mathrm{A})) \widetilde{\subset} \operatorname{sscl}((\mathrm{F}, \mathrm{A})) \widetilde{\cap} \operatorname{spcl}((\mathrm{F}, \mathrm{A}))$.

(ii) $\quad \operatorname{sbint}((\mathrm{F}, \mathrm{A})) \widetilde{\supset} \operatorname{sint}((\mathrm{F}, \mathrm{A})) \widetilde{\cup} \operatorname{spint}((\mathrm{F}, \mathrm{A}))$.

Proof (i) $\operatorname{sscl}((\mathrm{F}, \mathrm{A})) \widetilde{\cap} \operatorname{spcl}((\mathrm{F}, \mathrm{A})) \widetilde{\supset}[(F, A) \widetilde{\cup} \operatorname{int}(\operatorname{cl}((F$, $A)))] \widetilde{\cap}[(F, A) \widetilde{\cup} \operatorname{cl}(\operatorname{int}((\mathrm{F}, \mathrm{A})))]$

$=(F, A) \widetilde{\cup}[\operatorname{int}(\operatorname{cl}((\mathrm{F}, \mathrm{A}))) \widetilde{n} \operatorname{cl}(\operatorname{int}((\mathrm{F}, \mathrm{A})))]=\operatorname{sbcl}((\mathrm{F}, \mathrm{A}))$.

(ii) $\operatorname{ssint}((\mathrm{F}, \mathrm{A})) \widetilde{\cup} \operatorname{spint}((\mathrm{F}, \mathrm{A})) \quad \widetilde{\subset}[(F, A) \widetilde{\cap} \operatorname{cl}(\operatorname{int}((\mathrm{F}$, $A)))] \tilde{\cup}[(F, A) \widetilde{\cap} \operatorname{int}(\operatorname{cl}((\mathrm{F}, \mathrm{A})))]=(F, A) \widetilde{\cap}[\operatorname{cl}(\operatorname{int}((\mathrm{F}, \mathrm{A})))$ $\widetilde{U} \operatorname{int}(\operatorname{cl}((\mathrm{F}, \mathrm{A})))]=\operatorname{sbint}((\mathrm{F}, \mathrm{A}))$.

Theorem 17 Let $X$ be a soft topological space. If $(F, B)$ is an soft open set and $(F, A)$ is a sb-open set in $X$. Then $(F, A) \widetilde{\cap}(F, B)$ is a sb-open set in $X$. 
Proof

$(F, B) \widetilde{\cap}(F, A) \widetilde{C}(F, B) \widetilde{\cap}[\operatorname{cl}(\operatorname{int}((\mathrm{F}, \mathrm{A}))) \widetilde{\cup} \operatorname{int}(\operatorname{cl}((\mathrm{F}, \mathrm{A})))]$

$=[(F, B) \widetilde{n} \operatorname{cl}(\operatorname{int}((\mathrm{F}, \mathrm{A})))] \widetilde{\cup}[(\mathrm{F}, \mathrm{B}) \widetilde{\cap} \operatorname{int}(\operatorname{cl}((\mathrm{F}, \mathrm{A})))]$

$=[(\operatorname{cl}(\operatorname{int}((F, B))) \tilde{n} \operatorname{cl}(\operatorname{int}((F, A)))) \widetilde{\cup}(\operatorname{int}(\operatorname{cl}((F, B))) \widetilde{n} \operatorname{int}(\operatorname{cl}((F, A))))]$

$=[\operatorname{cl}(\operatorname{int}((F, B) \widetilde{\cap}(F, A))) \widetilde{\cup i n t}(\operatorname{cl}((F, B) \widetilde{\cap}(F, A)))]$.

Hence $(F, A) \widetilde{\cap}(F, B)$ is a sb-open set in $X$.

Theorem 18 Let $X$ be soft topological space. If $(F, B)$ is an $\mathrm{s} \alpha$-open set and $(F, A)$ is a sb-open set in $X$. Then $(F, A) \widetilde{\cap}(F, B)$ is a sb-open set in $X$.

\section{Proof}

$(F, B) \widetilde{\cap}(F, A) \widetilde{\subset} \operatorname{int}(\operatorname{cl}(\operatorname{int}((\mathrm{F}, \mathrm{B})))) \widetilde{\cap}[\operatorname{cl}(\operatorname{int}((\mathrm{F}, \mathrm{A}))) \widetilde{\cup} \operatorname{int}(\mathrm{cl}((\mathrm{F}, \mathrm{A})))]$

$=[\operatorname{int}(\mathrm{cl}(\operatorname{int}((\mathrm{F}, \mathrm{B})))) \tilde{n} \mathrm{cl}(\operatorname{int}((\mathrm{F}, \mathrm{A})))]$

$\widetilde{\cup}[\operatorname{int}(\operatorname{cl}(\operatorname{int}((F, B)))) \widetilde{n i n t}(\operatorname{cl}((F, A)))]$.

$\operatorname{int}(\operatorname{cl}((\mathrm{F}, \mathrm{A}))) \widetilde{C}[\operatorname{cl}(\operatorname{int}((\mathrm{F}, \mathrm{B})) \widetilde{\cap} \operatorname{cl}(\operatorname{int}((\mathrm{F}, \mathrm{A}))]$

$\widetilde{U}[\operatorname{int}(\operatorname{cl}((\mathrm{F}, \mathrm{B}))) \tilde{\cap} \operatorname{int}(\mathrm{cl}((\mathrm{F}, \mathrm{A})))]$

$\widetilde{C}[\operatorname{cl}(\operatorname{int}((\mathrm{F}, \mathrm{A}) \widetilde{\cap}(\mathrm{F}, \mathrm{B})))] \widetilde{\cup}[\operatorname{int}(\operatorname{cl}((\mathrm{F}, \mathrm{A}) \widetilde{\cap}(\mathrm{F}, \mathrm{B})))]$.

Hence $(F, A) \widetilde{\cap}(F, B)$ is sb-open set in $X$.

Theorem $19(B, E)$ is sb-open (sb-closed) set in $X$ if and only if $(B, E)$ is the union (intersection) of ss-open set and sb-open set in $X$.

Proof Follows from the Definitions 11.

\section{Soft b-continuity}

In this section, we introduce soft b-continuous maps, soft b-irresolute maps, soft b-closed maps and soft b-open maps and study some of their properties.

Definition 14 ([13]) let $(X, E)$ and $(Y, K)$ be soft classes. Let $u: X \rightarrow Y$ and $p: E \rightarrow K$ be mappings. Then a mapping $f:(X, E) \rightarrow(Y, K)$ is defined as: for a soft set $(F, A)$ in $(X, E),(f(F, A), B), B=p(A) \subseteq K$ is a soft set in $(Y, K)$ given by $f(F, A)(\beta)=u\left(\underset{\alpha \in p^{-1}(\beta) \cap A}{\cup F(\alpha)}\right)$ for $\beta \in K$. $(f(F, A), B)$ is called a soft image of a soft set $(F, A)$. If $B=K$, then we shall write $(f(F, A), K)$ as $f(F, A)$.

Definition 15 [13] Let $f:(X, E) \rightarrow(Y, K)$ be a mapping from a soft class $(X, E)$ to another soft class $(Y, K)$, and $(G, C)$ a soft set in soft class $(Y, K)$, where $C \subseteq K$. Let $u: X \rightarrow Y$ and $p: E \rightarrow K$ be mappings. Then $\left(f^{-1}(G, C), D\right), D=p^{-1}(C)$, is a soft set in the soft classes $(X, E)$, defined as: $f^{-1}(G, C)(\alpha)=u^{-1}(G(p(\alpha)))$ for $\alpha \in$ $D \subseteq E .\left(f^{-1}(G, C), D\right)$ is called a soft inverse image of $(G, C)$. Hereafter, we shall write $\left(f^{-1}(G, C), E\right)$ as $f^{-1}(G, C)$.
Theorem 20 [13] Let $f:(X, E) \rightarrow(Y, K), u: X \rightarrow Y$ and $p: E \rightarrow K$ be mappings. Then for soft sets $(F, A),(G, B)$ and a family of soft sets $\left(F_{i}, A_{i}\right)$ in the soft class $(X, E)$, we have:

(1) $f(\Phi)=\Phi$,

(2) $f(\tilde{X})=\tilde{Y}$,

(3) $f((F, A) \tilde{\cup}(G, B))=f(F, A) \tilde{\cup} f(G, B)$ in general $f\left(\cup_{i}\left(F_{i}, A_{i}\right)\right)=\cup_{i} f\left(F_{i}, A_{i}\right)$,

(4) $f((F, A) \tilde{\cap}(G, B)) \widetilde{\simeq} f(F, A) \tilde{\cap} f(G, B)$ in general $f\left(\cap_{i}\left(F_{i}, A_{i}\right)\right) \stackrel{\sim}{\subseteq} \cap_{i} f\left(F_{i}, A_{i}\right)$,

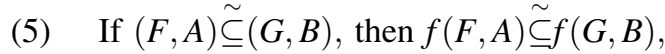

(6) $f^{-1}(\Phi)=\Phi$,

(7) $f^{-1}(\tilde{Y})=\tilde{X}$,

$f^{-1}((F, A) \tilde{\cup}(G, B))=f^{-1}(F, A) \tilde{\cup} f^{-1}(G, B) \quad$ in general $f^{-1}\left(\widetilde{\cup}_{i}\left(F_{i}, A_{i}\right)\right)=\widetilde{\cup}_{i} f^{-1}\left(F_{i}, A_{i}\right)$, $f^{-1}((F, A) \tilde{\cap}(G, B))=f^{-1}(F, A) \tilde{\cap} f^{-1}(G, B) \quad$ in general $f^{-1}\left(\widetilde{\cap}_{i}\left(F_{i}, A_{i}\right)\right)=\widetilde{\cap}_{i} f^{-1}\left(F_{i}, A_{i}\right)$,

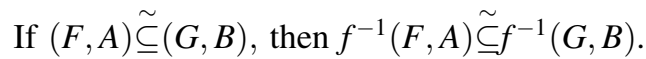

Definition 16 A soft mapping $f: X \rightarrow Y$ is said to be soft b-continuous (briefly sb-continuous) if the inverse image of each soft open set of $Y$ is a sb-open set in $X$.

Theorem 21 Let $f: X \rightarrow Y$ be a mapping from a soft space $X$ to soft space $Y$. Then, the following statements are true;

(i) $f$ is sb-continuous,

(ii) the inverse image of each soft closed set in $Y$ is soft b-closed in $X$.

Proof (i) $\Rightarrow$ (ii): Let $(G, K)$ be a soft closed set in $Y$. Then $(G, K)^{c}$ is soft open set. Thus, $f^{-1}\left((G, K)^{c}\right) \in \operatorname{SbOS}(\mathrm{X})$, i.e., $X-f^{-1}((G, K)) \in \operatorname{SbOS}(\mathrm{X})$. Hence $f^{-1}((G, K))$ is a sb-closed set in $X$.

(ii) $\Rightarrow(\mathrm{i})$ : Let $(O, K)$ is soft open set in $Y$. Then $(O, K)^{c}$ is soft closed set and by (ii) we have $f^{-1}\left((O, K)^{c}\right) \in \operatorname{SbCS}(\mathrm{X})$, i.e., $X-f^{-1}((O, K)) \in \operatorname{SbCS}(\mathrm{X})$. Hence $f^{-1}((O, K))$ is a sbopen set in $X$. Therefore, $f$ is a sb-continuous function.

Definition 17 A soft mapping $f: X \rightarrow Y$ is called soft $\beta$ continuous [9] (resp. soft $\alpha$-continuous [6], soft pre-continuous [6], soft semi-continuous [8]) if the inverse image of each soft open set in $Y$ is $\mathrm{s} \beta$-open (resp. s $\alpha$-open, spopen, ss-open) set in $X$.

Remark 3 We have following implications, however, examples given below show that the converses of these implications are not true. 
soft continity

soft $\alpha$-continity

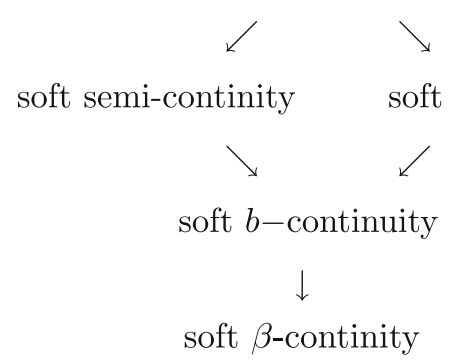

Example $3 f$ is a soft $\alpha$-continuous function, but not soft continuous function given Example 4 in [6].

Example $4 f$ is a soft pre-continuous function and soft, but not soft $\alpha$-continuous function given Example 5 in [6].

Example $5 f$ is a soft semi-continuous function and soft, but not soft $\alpha$-continuous function given Example 6 in [6].

Example 6 Let $X=\left\{h_{1}, h_{2}, h_{3}, h_{4}\right\}, Y=\left\{y_{1}, y_{2}, y_{3}, y_{4}\right\}$, $E=\left\{e_{1}, e_{2}, e_{3}\right\}$, and $K=\left\{k_{1}, k_{2}, k_{3}\right\}$ and let $(X, \tau, E)$ and $(Y, v, K)$ be soft topological spaces.

Define $u: X \rightarrow Y$ and $p: E \rightarrow K$ as

$u\left(h_{1}\right)=\left\{y_{1}\right\}, u\left(h_{2}\right)=\left\{y_{3}\right\}, u\left(h_{3}\right)=\left\{y_{2}\right\}, u\left(h_{4}\right)=\left\{y_{4}\right\}$, $p\left(e_{1}\right)=\left\{k_{2}\right\}, p\left(e_{2}\right)=\left\{k_{1}\right\}, p\left(e_{3}\right)=\left\{k_{3}\right\}$.

Let us consider the soft topology $\tau$ on $X$ given in Example 1; i.e.,

$$
\begin{aligned}
& \tau=\left\{\Phi, \tilde{X},\left(F_{1}, E\right),\left(F_{2}, E\right),\left(F_{3}, E\right), \ldots\left(F_{15}, E\right)\right\}, \\
& v=\{\Phi, \tilde{Y},(L, K)\} ; \\
& \quad(L, K)=\left\{\left(k_{1},\left\{y_{2}, y_{4}\right\}\right),\left(k_{2},\left\{y_{1}, y_{3}\right\}\right),\left(k_{3},\left\{y_{1}, y_{2}, y_{4}\right\}\right)\right\}
\end{aligned}
$$
and mapping;

$f:(X, \tau, E) \rightarrow(Y, v, K)$ is a soft mapping. Then $(L, K)$ is a soft open set in $Y ; f^{-1}((L, K))=$ $\left\{\left(e_{1},\left\{h_{1}, h_{2}\right\}\right),\left(e_{2},\left\{h_{3}, h_{4}\right\}\right),\left(e_{3},\left\{h_{1}, h_{3}, h_{4}\right\}\right)\right\}$ is a $\mathrm{s} \beta$ open set but not sb-open set in $X$. Therefore, $f$ is a soft $\beta$-continuous function but not sb-continuous function.

Example 7 Let $X=\left\{h_{1}, h_{2}, h_{3}, h_{4}\right\}, Y=\left\{y_{1}, y_{2}, y_{3}, y_{4}\right\}$, $E=\left\{e_{1}, e_{2}, e_{3}\right\}$, and $K=\left\{k_{1}, k_{2}, k_{3}\right\}$ and let $(X, \tau, E)$ and $(Y, v, K)$ be soft topological spaces.

Define $u: X \rightarrow Y$ and $p: E \rightarrow K$ as

$u\left(h_{1}\right)=\left\{y_{1}\right\}, u\left(h_{2}\right)=\left\{y_{3}\right\}, u\left(h_{3}\right)=\left\{y_{2}\right\}, u\left(h_{4}\right)=\left\{y_{4}\right\}$, $p\left(e_{1}\right)=\left\{k_{2}\right\}, p\left(e_{2}\right)=\left\{k_{1}\right\}, p\left(e_{3}\right)=\left\{k_{3}\right\}$.

Let us consider the soft topology $\tau$ on $X$ given in Example 1; i.e.,

$$
\begin{gathered}
\tau=\left\{\Phi, \tilde{X},\left(F_{1}, E\right),\left(F_{2}, E\right),\left(F_{3}, E\right), \ldots\left(F_{15}, E\right)\right\}, \\
v=\{\Phi, \tilde{Y},(M, K)\} ; \\
\quad(M, K)=\left\{\left(k_{1},\left\{y_{1}, y_{2}, y_{3}\right\}\right),\left(k_{2},\left\{y_{4}\right\}\right),\left(k_{3},\left\{y_{3}, y_{4}\right\}\right)\right\}
\end{gathered}
$$$$
\text { and mapping; }
$$

$f:(X, \tau, E) \rightarrow(Y, v, K)$ is a soft mapping. Then $(M, K)$ is a soft open set in $Y ; f^{-1}((M, K))=$ $\left\{\left(e_{1},\left\{h_{4}\right\}\right),\left(e_{2},\left\{h_{1}, h_{2}, h_{3}\right\}\right),\left(e_{3},\left\{h_{2}, h_{4}\right\}\right)\right\}$ is a sb-open set but not ss-open set in $X$. Hence, $f$ is a $s b$-continuous function but not soft semi-continuous function.

Example 8 Let $X=\left\{h_{1}, h_{2}, h_{3}, h_{4}\right\}, Y=\left\{y_{1}, y_{2}, y_{3}, y_{4}\right\}$, $E=\left\{e_{1}, e_{2}, e_{3}\right\}$, and $K=\left\{k_{1}, k_{2}, k_{3}\right\}$ and let $(X, \tau, E)$ and $(Y, v, K)$ be soft topological spaces.

Define $u: X \rightarrow Y$ and $p: E \rightarrow K$ as

$u\left(h_{1}\right)=\left\{y_{1}\right\}, u\left(h_{2}\right)=\left\{y_{3}\right\}, u\left(h_{3}\right)=\left\{y_{2}\right\}, u\left(h_{4}\right)=\left\{y_{4}\right\}$, $p\left(e_{1}\right)=\left\{k_{2}\right\}, p\left(e_{2}\right)=\left\{k_{1}\right\}, p\left(e_{3}\right)=\left\{k_{3}\right\}$.

Let us consider the soft topology $\tau$ on $X$ given in Example 1; i.e.,

$$
\begin{gathered}
\tau=\left\{\Phi, \tilde{X},\left(F_{1}, E\right),\left(F_{2}, E\right),\left(F_{3}, E\right), \ldots\left(F_{15}, E\right)\right\}, \\
v=\{\Phi, \tilde{Y},(N, K)\} ; \\
(N, K)=\left\{\left(k_{1},\left\{y_{1}, y_{2}\right\}\right),\left(k_{2},\left\{y_{3}, y_{4}\right\}\right),\left(k_{3},\left\{y_{1}, y_{2}, y_{4}\right\}\right)\right\}
\end{gathered}
$$
and mapping;

$f:(X, \tau, E) \rightarrow(Y, v, K)$ is a soft mapping. Then $(N, K)$ is a soft open set in $Y ; f^{-1}((N, K))=\left\{\left(e_{1},\left\{h_{2}, h_{4}\right\}\right)\right.$, $\left.\left(e_{2},\left\{h_{1}, h_{3}\right\}\right),\left(e_{3},\left\{h_{1}, h_{3}, h_{4}\right\}\right)\right\}$ is a sb-open set but not spopen set in $X$. Thus, $f$ is a sb-continuous function, but not soft pre-continuous function.

Theorem 22 Every soft continuous function is sb-continuous function.

Proof Let $f: X \rightarrow Y$ be a soft continuous function. Let $(F, K)$ be a soft open set in $Y$. Since $f$ is soft continuous, $f^{-1}((F, K))$ is soft open in $X$. And so $f^{-1}((F, K))$ is sbopen set in $X$. Therefore, $f$ is sb-continuous function.

Definition 18 A mapping $f: X \rightarrow Y$ is said to be soft b-irresolute (briefly sb-irresolute) if $f^{-1}((F, K))$ is sbclosed set in $X$, for every sb-closed set $(F, K)$ in $Y$.

Theorem 23 A mapping $f: X \rightarrow Y$ is sb-irresolute mapping if and only if the inverse image of every sb-open set in $Y$ is sb-open set in $X$.

Theorem 24 Every sb-irresolute mapping is sb-continuous mapping.

Proof Let $f: X \rightarrow Y$ is sb-irresolute mapping. Let $(F, K)$ be a soft closed set in $Y$, then $(F, K)$ is sb-closed set in $Y$. Since $f$ is sb-irresolute mapping, $f^{-1}((F, K))$ is a sb-closed set in $X$. Hence, $f$ is sb-continuous mapping.

Theorem 25 Let $f:(X, \tau, E) \rightarrow(Y, v, K), \quad g:$ $(Y, v, K) \rightarrow(Z, \sigma, T)$ be two functions. Then

(i) $g \circ f: X \rightarrow Z$ is sb-continuous, if $f$ is sb-continuous and $g$ is soft continuous.

(ii) $g \circ f: X \rightarrow Z$ is sb-irresolute, if $f$ and $g$ is sbirresolute functions. 
(iii) $g \circ f: X \rightarrow Z$ is sb-continuous if $f$ is sb-irresolute and $g$ is sb-continuous.

Proof

(i) Let $(H, T)$ be soft closed set of $Z$. Since $g: Y, \rightarrow$ $Z$ is soft continuous, by definition $g^{-1}((H, T))$ is soft closed set of $Y$. Now $f: X \rightarrow Y$ is sbcontinuous and $g^{-1}((H, T))$ is soft closed set of $Y$, so by Definition $16, f^{-1}\left(g^{-1}((H, T))\right)=(g \circ$ $f)^{-1}((H, T))$ is sb-closed in $X$. Hence $g \circ f: X \rightarrow$ $Z$ is sb-continuous.

(ii) Let $g: Y \rightarrow Z$ is sb-irresolute and let $(H, T)$ be sbclosed set of $Z$. Since $g$ is sb-irresolute by Definition $18, g^{-1}((H, T))$ is sb-closed set of $Y$. Also $f: X \rightarrow Y$ is sb-irresolute, so $f^{-1}\left(g^{-1}((H, T))\right)=(g \circ f)^{-1}((H, T)) \quad$ is $\quad$ sbclosed. Thus, $g \circ f: X \rightarrow Z$ is sb-irresolute.

(iii) Let $(H, T)$ be sb-closed set of $Z$. Since $g: Y \rightarrow Z$ is sb-continuous, $g^{-1}((H, T))$ is sb-closed set of $Y$. Also $f: X \rightarrow Y$ is sb-irresolute, so every sb-closed set of $Y$ is $s b$-closed in $X$. Therefore, $f^{-1}\left(g^{-1}((H, T))\right)=(g \circ f)^{-1}((H, T))$ is sb-closed set of $X$. Thus, $g \circ f: X \rightarrow Z$ is sb-continuous.

Theorem 26 If the bijective map $f: X \rightarrow Y$ is soft open and sb-irresolute, then $f$ is sb-irresolute.

Proof Let $(F, K)$ be a sb-closed set in $Y$ and let $f^{-1}((F, K)) \widetilde{\subset}(F, A)$ where $(F, A)$ is a soft open set in $X$. Clearly, $(F, K) \widetilde{\subset} f(F, A)$. Since $f: X \rightarrow Y$ is soft open map, by definition $f((F, A))$ is soft open in $Y$ and $(F, K)$ is sb-closed set in $Y$. Then $\operatorname{sbcl}((\mathrm{F}, \mathrm{K})) \widetilde{\subset} \mathrm{f}((\mathrm{F}, \mathrm{A}))$, and hence $f^{-1}(\operatorname{sbcl}((\mathrm{F}, \mathrm{K}))) \widetilde{\subset}(\mathrm{F}, \mathrm{A})$. Also $f$ is sb-irresolute map and $\operatorname{sbcl}((\mathrm{F}, \mathrm{K}))$ is a sb-closed set in $Y$, then $f^{-1}(\operatorname{sbcl}((\mathrm{F}, \mathrm{K})))$ is sb-closed set in $X$. Thus, $\operatorname{sbcl}\left(\mathrm{f}^{-1}((F, K))\right)$ $\widetilde{\subset} \operatorname{sbcl}\left(\mathrm{f}^{-1}(\operatorname{sbcl}((\mathrm{F}, \mathrm{K})))\right) \widetilde{\subset}(\mathrm{F}, \mathrm{A})$. So $f^{-1}((F, K))$ is sb-closed set in $X$. Therefore, $f: X \rightarrow Y$ is sb-irresolute map.

Definition 19 A mapping $f: X \rightarrow Y$ is said to be soft b-open (briefly sb-open) map if the image of every soft open set in $X$ is sb-open set in $Y$.

Definition 20 A mapping $f: X \rightarrow Y$ is said to be soft b-closed (briefly sb-closed) map if the image of every soft closed set in $X$ is sb-closed set in $Y$.

Theorem 27 If $f: X \rightarrow Y$ is soft closed function and $g$ : $Y \rightarrow Z$ is sb-closed function, then $g \circ f$ is sb-closed function.

Proof For a soft closed set $(F, A)$ in $X, f((F, A))$ is soft closed set in $Y$. Since $g: Y \rightarrow Z$ is sb-closed function,
$g(f((F, A)))$ is sb-closed set in Z. $g(f((F, A)))=(g \circ$ $f)((F, A))$ is sb-closed set in $Z$. Therefore, $g \circ f$ is sbclosed function.

Theorem 28 A map $f: X \rightarrow Y$ is sb-closed if and only if for each soft set $(F, K)$ of $Y$ and for each soft open set $(F, A)$ such that $f^{-1}((F, K)) \widetilde{\subset}(F, A)$, there is a sb-open set $(G, K)$ of $Y$ such that $(F, K) \widetilde{\subset}(G, K)$ and $f^{-1}(G, K)$ $\widetilde{\subset}(F, A)$.

Proof Suppose $f$ is sb-closed map. Let $(F, K)$ be a soft set of $Y$, and $(F, A)$ be a soft open set of $X$, such that $f^{-1}((F, K)) \widetilde{\subset}(F, A)$. Then $(G, K)=\left(f\left((F, A)^{c}\right)\right)^{c}$ is a sbopen set in $Y$ such that $(F, K) \widetilde{\subset}(G, K)$ and $f^{-1}((G, K))$ $\widetilde{\subset}(F, A)$.

Conversely, suppose that $(F, B)$ is a soft closed set of $X$. Then $f^{-1}\left((f((F, B)))^{c}\right) \widetilde{C}(F, B)^{c}$, and $(F, B)^{c}$ is soft open set. By hypothesis, there is a sb-open set $(G, K)$ of $Y$ such that $\left(f\left((F, A)^{c}\right)^{c} \widetilde{\subset}(G, K)\right.$ and $f^{-1}((G, K)) \widetilde{\subset}(F, B)$. Thus $(F, B) \widetilde{\subset} f^{-1}((G, K))$. Hence $(G, K)^{c} \widetilde{\subset} f((G, K)) \widetilde{\subset} f\left(\left(f^{-1}\right.\right.$ $\left.((G, K)))^{c}\right) \widetilde{\subset}(G, K)$, which implies $f((F, B))=(G, K)^{c}$. Since $(G, K)^{c}$ is sb-closed set, $f((F, B))$ is sb-closed set. So $f$ is a sb-closed map.

Theorem 29 Let $f: X \rightarrow Y, g: Y \rightarrow Z$ be two maps such that $g \circ f: X \rightarrow Z$ is sb-closed map.

(i) If $f$ is soft continuous and surjective, then $g$ is sbclosed map.

(ii) If $g$ is sb-irresolute and injective, then $f$ is sb-closed map.

\section{Proof}

(i) Let $(H, K)$ be a soft closed set of $Y$. Then, $f^{-1}((H, K))$ is soft closed set in $X$ as $f$ is soft continuous. Since $g \circ f$ is sb-closed map, $(g \circ$ $f)\left(f^{-1}((H, K))\right)=g((H, K))$ is sb-closed set in $Z$. Hence $g: Y \rightarrow Z$ sb-closed map.

(ii) Let $(H, E)$ be a soft closed set in $X$. Then, $(g \circ$ $f)((H, E))$ is sb-closed set in $Z$, and so $g^{-1}(g \circ$ $f)((H, E))=f((H, E))$ is sb-closed set in $Y$. Since $g$ is sb-irresolute and injective. Hence $f$ is a sbclosed map.

Theorem 30 If $(F, B)$ is sb-closed set in $X$ and $f: X \rightarrow Y$ is bijective, soft continuous and sb-closed, then $f((F, B))$ is sb-closed set in $Y$.

Proof Let $f((F, B)) \widetilde{\subset}(F, K)$ where $(F, K)$ is a soft open set in $Y$.

Since $f$ is soft continuous, $f^{-1}((F, K))$ is a soft open set containing $(F, B)$. 
Hence $\operatorname{sbcl}((\mathrm{F}, \mathrm{B})) \widetilde{\subset} \mathrm{f}^{-1}((\mathrm{~F}, \mathrm{~K}))$ as $(F, B)$ is sb-closed set.

Since $f$ is sb-closed, $f(\operatorname{sbcl}((\mathrm{F}, \mathrm{B})))$ is sb-closed set contained in the soft open set $(F, K)$,

which implies $\operatorname{sbcl}(\mathrm{f}(\operatorname{sbcl}((\mathrm{F}, \mathrm{B})))) \widetilde{C}(\mathrm{~F}, \mathrm{~K})$ and hence $\operatorname{sbcl}(\mathrm{f}((\mathrm{F}, \mathrm{B}))) \widetilde{C}(\mathrm{~F}, \mathrm{~K})$. So $f((F, B))$ is sb-closed set in $Y$.

\section{Conclusion}

In this paper, we introduce the concept of soft b-open sets and soft $\mathrm{b}$-continuous functions in topological spaces and some of their properties are studied. We also introduce soft $\mathrm{b}$-interior and soft b-closure and have established several interesting properties. In the end, we hope that this paper is just a beginning of a new structure, it will be necessary to carry out more theoretical research to promote a general framework for the practical application.

Open Access This article is distributed under the terms of the Creative Commons Attribution License which permits any use, distribution, and reproduction in any medium, provided the original author(s) and the source are credited.

\section{References}

1. Molodtsov, D.: Soft set theory-First results. Comput. Math. Appl. 37(4-5), 19-31 (1999)
2. Shabir, M., Naz, M.: On soft topological spaces. Comput. Math. Appl. 61, 1786-1799 (2011)

3. Zorlutuna, I., Akdag, M., Min, W.K., Atmaca, S.: Remarks on soft topological spaces. Ann. Fuzzy Math. Inf. 3(2), 171-185 (2012)

4. Aygunoglu, A., Aygun, H: Some notes on soft topological spaces. Neural Comput. Appl. doi:10.1007/s00521-011-0722-3

5. Chen, B.: Soft semi-open sets and related properties in soft topological spaces. Appl. Math. Inf. Sci. 7(1), 287-294 (2013)

6. Akdag, M., Ozkan, A: Soft $\alpha$-open sets and soft $\alpha$-continuous functions. Abstr. Anal. Appl. Art ID 891341, 1-7 (2014)

7. Aras, C.G., Sonmez, A., Cakall, H: On soft mappings. http:// arxiv.org/abs/1305.4545 (Submitted)

8. Mahanta, J., Das, PK: On soft topological space via semiopen and semiclosed soft sets. http://arxiv.org/abs/1203.4133 (Submitted)

9. Yumak, Y., Kaymakc1, AK: Soft $\beta$-open sets and their aplications. http://arxiv.org/abs/1312.6964 (Submitted)

10. Maji, P.K., Biswas, R., Roy, A.R.: Soft set theory. Comput. Math. Appl. 45, 555-562 (2003)

11. Ali, M.I., Feng, F., Liu, X., Min, W.K., Shabir, M.: On some new operations in soft set theory. Comput. Math. Appl. 57, 1547-1553 (2009)

12. Arockiarani, I., Arokialancy, A.: Generalized soft $\mathrm{g} \beta$-closed sets and soft gs $\beta$-closed sets in soft topological spaces. Int. J. Math. Arch. 4(2), 1-7 (2013)

13. Kharal, A., Ahmad, B.: Mappings on soft classes. New Math. Nat. Comput. 7(3), 471-481 (2011) 